\title{
TENSIONES JURÍDICAS RESPECTO DE LOS MECANISMOS DE REFRENDACIÓN E IMPLEMENTACIÓN DEL ACUERDO FINAL ENTRE GOBIERNO Y FARC-EP PARA LA TERMINACIÓN DEL CONFLICTO \\ Legal tensions regarding the mechanisms of endorsement and implementation of the final agreement between government and FARC-EP for the termination of the conflict
}

Jorge Mejia Turizo $^{1}$ Juan Carlos Berrocal Durán ${ }^{2}$

Fecha de recepción: 22 de abril de 2017

Fecha de aceptación: 04 de mayo de 2017

Sumario: 1. Introducción; 2. Metodología; 3. Resultados; 3.1. En lo atinente al mecanismo de refrendación; 3.2. En lo atinente al mecanismo de implementación; 4. Conclusiones. 5. Referencias Bibliográficas.

\footnotetext{
${ }^{1}$ Abogado, Magister en Derecho administrativo, Doctorando en Derecho, Ciencia política y Criminología por la Universidad de Valencia, España. Diplomado en Gestión y Públicas de la Escuela Superior de administración Pública ESAP, Docente investigador del grupo de investigación Andrés Bello de Corporación Universitaria Rafael Núñez, Campus Barranquilla., Ex becario del programa jóvenes investigadores e innovadores de Colciencias. Correo electrónico: Jorge.mejia@curnvirtual.edu.co

${ }^{2}$ Abogado. Magíster en Derecho Procesal y especialista en Derecho Laboral. Candidato a Doctor en Ciencia Política, Docente investigador del grupo de investigación Andrés Bello de Corporación Universitaria Rafael Núñez, Campus Barranquilla. Correo electrónico: juan.berrocal@curnvirtual.edu.co
} 


\section{CóMO SE CITA ESTE ARTícuLO (Normas APA-6)}

Mejia Turizo, Jorge \& Berrocal Durán, Juan Carlos (2017). Tensiones jurídicas respecto de los mecanismos de refrendación e implementación del acuerdo final entre gobierno y FARC-EP para la terminación del conflicto. Revista Jurídica Mario Alario D’Filippo, IX (18), pág 111-124.

\section{RESUMEN}

El artículo que se acomete a continuación tiene como propósitos centrales hacer un análisis descriptivo y crítico de los mecanismos de refrendación e implementación que se a llevaron a cabo en el marco del "acuerdo final para la terminación del conflicto y la construcción de una paz estable y duradera entre el Gobierno y las Farc-EP". Para lo cual se analizará la refrendación desde el punto de vista de la idoneidad del mecanismo escogido para consultar al pueblo, y en cuanto a la implementación se abordará desde el enfoque de producción normativa y desde el enfoque el material de ejecución, la pertinencia de los mecanismos de implementación escogidos en términos de legitimidad, eficacia y conveniencia.

\section{PALABRAS CLAVE}

Refrendación, implementación, acuerdo para terminación de conflicto, mecanismos de participación ciudadana.

\section{ABSTRACT}

The main purpose of this article is to make a descriptive and critical analysis of the mechanisms of referendum and implementation that were carried out in the framework of the "final agreement for the ending of the conflict and the construction of a stable and lasting peace between the Government and the Farc-EP". For this purpose, the referendum issue will be analyzed from the point of view of the suitability of the chosen mechanism to consult the people, while the issue of implementation will be addressed from the perspective of normative production, material of execution, pertinence of the chosen implementation mechanisms in terms of legitimacy, effectiveness and convenience.

\section{KEYWORDS}

Endorsement, implementation, agreement for termination of conflict, citizen participation mechanisms. 


\section{INTRODUCCIÓN}

En el presente artículo se planteará desde un análisis crítico y ponderado la tensión que se ha suscitado a raíz de la escogencia de un mecanismo que permita conocer los niveles de aprobación popular de los "acuerdos para la terminación del conflicto y la construcción de una paz estable y duradera" entre Gobierno y FARC-EP. Esta tensión se presentó justamente, por la falta de unificación de criterios para determinar cuál de los mecanismos de participación ciudadana contemplados en la constitución política era en su momento el más idóneo para llevar a cabo la refrendación, o si se podría utilizar un mecanismo sui generis distintos de los consagrados en el actual derecho positivo nacional, o simplemente si no es necesaria la realización de un dispositivo instrumental de consulta. Aunque la Corte constitucional colombiana ya adoptó una decisión en materia de la constitucionalidad del plebiscito como mecanismo para someter los acuerdos a un proceso de legitimación popular, que ya también se realizó, es significativo afrontar un estudio sopesado de las posturas académicas y políticas que discrepan de la virtud del mecanismo escogido para garantizar una real legitimidad democrática, asimismo es menester analizar las tesis que sostienen la desnaturalización del plebiscito por las nuevas esencias y características que se le dotaron para eseproceso refrendatorio en particular.

Hay que destacar adicionalmente, que en este artículo se desarrollará una reflexión aguda sobre las teorías que conciben el mecanismo refrendatorio del plebiscito como una forma de sustitución de la constitución, por encontrarse desbordando su órbita de competencia el legislador estatutario, o si por el contrario está actuando en el marco de la libertad de configuración legislativa.

Otro punto importante en que se centra está investigación es el referente a la "implementación" de los acuerdos para la terminación del conflicto ente el Gobierno y las FARC - EP, el cual será analizado desde dos enfoques o dos concepciones funcionales bien diferenciadas. El primer enfoque la constituirá el análisis de la "implementación" como un proceso de conversión de los acuerdos en normas jurídicas vinculantes con plena legitimidad constitucional, que se traduce que ha superado los trámites legislativos correspondientes. Asimismo este enfoque de la implementación incluye trámites administrativos de stricto carácter normativo como la expedición de decretos pertinentes, y la formulación de políticas públicas, planes y programas. Sea preciso mencionar, que para los efectos de este enfoque que señalamos, constituirán comisiones para constatar que el contenido de los proyecto de actos legislativos, de ley o de actos administrativos sean coincidente con los acuerdos suscritos por las partes sentadas en la mesa de negociaciones.

El segundo enfoque de implementación que se analizará, desborda las cuestiones relativas a la mera producción normativa sea por vía legislativa o administrativa, y se 
concretará en la parte material de actuación, esto es, todo el conjunto de actividades, acciones, desarrollos operacionales y logísticos que se deben llevar a cabo para poder en práctica el grueso de disposiciones jurídicas que previamente fueron el documento del acuerdo final. Es este aspecto concerniente a la parte operativa, es pertinente destacar que se establecerán unos organismos para realizar evaluación, seguimiento y medición del grado de cumplimiento de las normas derivadas de los acuerdos.

\section{METODOLOGÍA}

El presente proyecto se encuentra estructurado metodológicamente a partir de un enfoque de carácter cualitativo, usándose un nivel de investigación de corte descriptivo, además el método empleado fue deductivo, y el paradigma de investigación que se aplica para su desarrollo es el crítico social.

\section{RESULTADOS}

\subsection{EN LO ATINENTE AL MECANISMO DE REFRENDACIÓN}

En este punto del presente artículo nos centraremos en el aspecto de la refrendación de los acuerdos para la terminación del conflicto entre el Gobierno y las Farc-EP, señalando que este mecanismo se traduce en la posibilidad que se le da al pueblo para que manifieste su conformidad o no con lo concertado. Para Rodrigo Uprimy (2013), la refrendación es la ratificación democrática de un eventual acuerdo de paz. Por su parte, la Misión de observación electoral MOE (2013) considera que el término en cuestión hace alusión a un instrumento que determina y permite conocer si existe apoyo o aprobación popular para las decisiones y acciones que se deban desarrollar en la búsqueda de la terminación del conflicto. Ahora bien, algunos sectores de la academia y la institucionalidad colombiana consideran que este instrumento de legitimación democrática no era necesario. Tal postura es sostenida por ejemplo, en la Fiscalía general de la nación, cuando fue regentada hasta el primer cuatrimestre del año 2016 por Eduardo Montealegre Lynett, quien señalaba que "no se requiere desde el punto de vista legal o constitucional la refrendación para celebrar los acuerdos" (Revista Semana, Enero 2015), insistiendo que no hay necesidad de una refrendación popular. Asimismo, esta entidad con función acusadora, plantea la tesis fundamentada en que el derecho a la paz es de carácter fundamental y por tanto debe ser inmune a las decisiones de las mayorías, lo que presupone su naturaleza o su condición de ser contra-mayoritario. En ese mismo sentido, el jurista Armando Novoa (citado por ámbito jurídico, 2016), considera que "en el momento actual, los mecanismos de refrendación popular no eran viables. Ni en el mejor escenario va a ser posible una convalidación de los acuerdos de paz. El Congreso debería otorgarle facultades al Presidente de la República, para que adopte las medidas para llevar a buen puerto el proceso". 
Establecido el concepto de refrendación, es menester dilucidar cuál debió ser el mecanismo más idóneo para su materialización. Y éste justamente ha sido uno de los escollos más sobresaliente que ha emergido en el proceso para la terminación de conflicto con las FARC. Puesto que no había resultado fácil establecer consensos entre las diferentes vertientes políticas, y entre los actores sentados en la mesa de negociación a la hora de definir el mecanismo de refrendación. Con relación al instrumento que debió ser utilizando Uprimy (2013) destacó que "existen múltiples vías, pero es necesario determinar cuál o cuáles son las mejores. Colombia dispone de un abanico de mecanismos, que van de los menos participativos a aquellos que implican una intervención ciudadana robusta" Cierto es pues, que la legislación colombiana ha dispuesto de una amplia gana de herramientas que permiten que el pueblo participe en la toma de decisiones políticas, sociales, económicas o culturales del orden nacional, departamental, municipal, distrital o local. Tales instrumentos están consagrados en el artículo 103 de la constitución política y desarrollados por la leyes 134 de 1994 y 1757 de 2015.

Los mecanismos que establece la carta política para la participación ciudadana y que podían servir para la refrendación de los acuerdos de paz, son el plebiscito, el referendo, la consulta popular, y la consulta especial para convocar una asamblea constituyente, aunque cierto sector de la doctrina señala que los mecanismos de participación contenidos en el artículo 103 de la constitución no tienen un carácter taxativo sino enunciativo, y por lo tanto podrían crearse figuras sui generis para hacer efectivo la participación de ciudadanos en una determinada cuestión. Justamente en la sentencia de la Corte constitucional que define el mecanismos de refrendación para los acuerdos de terminación conflicto, numerada C-379 de 2016, hay varias intervenciones que coadyuvan esta postura (entre las que se resalta la de la Registraduría Nacional del Estado Civil y la del Ministerio del interior) y el alto tribunal la retoma cuando sostiene: "debe recordarse que la jurisprudencia constitucional ha dejado por sentado que los mecanismos de participación ciudadana no tienen carácter taxativo, puesto que ello sería contrario a la naturaleza expansiva y universal del principio democrático. Esto explica que el legislador estatutario pueda prever procedimientos de participación ciudadana, incluso novedosos respecto de los previstos en la Constitución, pues además de las citadas características del principio democrático, no existe ninguna cláusula en la Carta Política que le imponga una limitación en ese sentido"

Con relación al uso de estos mecanismos en América latina, Daniel Zovatto (2010) sostiene que a nivel comparado, hay una variedad de instituciones de democracia directa y una pluralidad conceptual y terminológica que podría generar confusión. Teniendo en cuenta que la mayoría de las constituciones de este continente denominan estos instrumentos con términos diferentes -iniciativa legislativa popular, plebiscito, referendo, consulta popular, revocatoria de mandato, cabildo abierto, para citar tan sólo algunas de las expresiones más usuales-, la búsqueda de una unidad de acepciones y conceptos que trasciendan el ámbito nacional resulta, aunque difícil, imprescindible para entender mejor cuando 
hablamos de este tema. Por su parte desde México, David Altman (2010) sostiene que de estos mecanismos emergen cierto peligro y estrés político, pues establecen un juego de suma cero, mediante el cual la mayoría lo gana todo y la minoría lo pierde todo; no hay posibilidad de concesiones respecto de los problemas presentados; y existe, además, un claro riesgo de tiranía de la mayoría sobre la minoría.

Volviendo al caso colombiano, es pertinente señalar que los mecanismos de participación ciudadana no registran una realización constante o periódica en el país, tal como lo sostiene el consejero Augusto Hernández Becerra (2003), al considerar que la participación del pueblo es limitada y prácticamente se reduce a la votación en los órganos de elección popular. Se puede contar por ejemplo el plebiscito de 1957 en que votaron por primera vez las mujeres y se decidió la instauración del frente nacional. También se puede destacar que a comienzos del año 1990 estudiantes de varias universidades del país promovieron la inclusión de una "séptima papeleta" en los sufragios del 11 de marzo de 1990 para apoyar la convocatoria a una asamblea constituyente, a pesar de que este dispositivo de reforma constitucional no estaba autorizado en la Constitución. En el año 2003, se propuso un referendo con 18 preguntas de considerable extensión, de las cuales solo una pregunta superó el umbral para ser aprobado.

De los diferentes mecanismos de participación que se han señalado precedentemente, los que desde el punto de vista técnico jurídico tuvieron la mayor posibilidad de ser utilizados para la refrendación de los acuerdos de paz con la FARC, son el plebiscito, el referendo y la consulta popular, por la característica que estos entrañan. Aunque algunos sectores como el Partido político Centro democrático y la misma organización guerrillera FARC en algún momento manifestaron que el mecanismos que más le parece conveniente es la convocatoria a una asamblea nacional constituyente. Posición con la que discrepamos en esta investigación, toda vez que este es un mecanismo para ajustar un nuevo diseño constitucional, prácticamente para renovar al Estado y no se requiere para refrendar unos acuerdos. Bajo esta misma tesitura, Ramiro Bejarano (2016), citando al pensador italiano Luigi Ferrajoli, sostiene que "la legitimidad de un acuerdo de paz, viene por la sola circunstancia de que haya paz".

Los mecanismos que tienen mayores condiciones, desde la técnica jurídica, para tener vocación de idóneo para para refrendación popular, son el plebiscito y el referendo. Teniendo en cuenta estas consideraciones, se tratará de circunscribir la discusión a cuál mecanismo entre el referendo y el plebiscito es el más viable e idóneo jurídicamente para Ilevar a cabo la refrendación, pese a que la Corte Constitucional mediante sentencia C-379 de 2016 ya ha zanjado la discusión, aun en el ambiente de la comunidad jurídica queda la sensación que faltan elementos importantes por dilucidar, que llevan a considerar por algunos que la solución dada por la alta corporación es ilegítima. 
De acuerdo con el artículo tercero de la ley 134 de 1994 el referendo es la convocatoria que se hace al pueblo para que apruebe o rechace un proyecto de norma jurídica o derogue o no una norma ya vigente. El referendo puede ser nacional, regional, departamental, distrital, municipal o local. Este mecanismo puede ser derogatorio o aprobatorio de acuerdo a si lo que se consulta es la derogación o aprobación de un acto legislativo, de una ley, de una ordenanza, de un acuerdo o de una resolución local. De otra parte, esta misma ley, establece que el plebiscito es el pronunciamiento del pueblo convocado por el Presidente de la República, mediante el cual apoya o rechaza una determinada decisión del Ejecutivo.

Teniendo de presente estas definiciones, a nuestro juicio el referendo tiene menos vocación de ser el mecanismo idóneo, si hiciéramos una ponderación frente al plebiscito. Ahora sostenemos que el referendo es el mecanismo menos adecuado, puesto que lo que se va a discutir no es un texto legal o constitucional, sino un acuerdo que no tiene connotación o condición de norma jurídica, aunque muchos de sus aspectos pueden ser avalados posteriormente como tales a través de la implementación. Pero si puede considerarse que la estructuración y el cumplimento de los acuerdos es del resorte del gobierno, y en ese sentido puede darse la circunstancia de ser una decisión de competencia del ejecutivo, lo cual habilita para que sea el plebiscito el más pertinente de los mecanismos, como efectivamente se usó.

Ahora bien, desde el punto de vista práctico, seria desaconsejable usar el referendo teniendo en cuenta que para éste se exige que el votante se manifieste punto por punto o artículo por artículo, lo que implicaría que tendría que hacérsele varias preguntas al votante, que convertiría a la consulta en un cuestionario complejo y tedioso. Además que esto pudo haber conllevado a que unas preguntas superen el umbral y otras no, lo que socavaría ciertas bases fundantes del acuerdo, como el principio que establecía que lo acordado tendría una vocación de integralidad o funcionaría como un cuerpo monolítico. La Corte Constitucional mediante sentencia C- 141 de 2010 ha prohibido en el referéndum el voto en bloque para los aspectos de contenido multi-temático, como perfectamente lo es los acuerdos de paz suscritos con las FARC. Pues bien, el mecanismo del plebiscito no establece esa limitante y bien puede establecerse en la papeleta una sola pregunta que se responda con un "SI" o un "NO", como efectivamente ha quedó planteado.

En lo que respecta al trámite, el del referendo es un poco más extenso porque implica la aprobación de una ley por parte del Congreso y la revisión de la Corte Constitucional. De acuerdo con León (2015) Podría tardar como mínimo 8 meses. En el plebiscito el presidente envía un informe al congreso, y si dentro del mes siguiente a la fecha en que el Presidente haya informado sobre su intención de realizar un plebiscito, ninguna de las dos Cámaras, por la mayoría de asistentes, haya manifestado su rechazo, el Presidente podrá convocarlo. La votación ciudadana no podrá no podrá ser anterior aun mes ni posterior 
a cuatro meses, contados a partir de la fecha en que el Congreso reciba el informe del Presidente. El plebiscito no requiere de revisión previa por la corte constitucional.

Así las cosas, un referendo hubiese implicado que se debía debatir en el congreso de la republica pregunta por pregunta, y posteriormente que la Corte Constitucional realice un análisis de constitucionalidad. Para después entregar un entramado de preguntas a la ciudadanía.

En lo que corresponde al umbral que se requiere para su aprobación la ley 1757 de 2015 señala que para el Referendo se necesita que el voto afirmativo de más de la mitad de los sufragantes y que el número de estos exceda de la cuarta $(25 \%)$ parte del total de ciudadanos que integran el censo electoral y para el plebiscito que haya participado más del cincuenta por ciento (50\%) del censo electoral vigente. Aclarando que La Corte Constitucional, en Sentencia C - 1121 de 2004, definió el censo electoral como "una base de datos donde se incluyen los ciudadanos colombianos, residentes en el país y en el exterior, que pueden válidamente sufragar en un determinado certamen democrático, y que le permite al Estado controlar, planear, organizar y desarrollar no sólo los certámenes electorales sino igualmente los mecanismos de participación".

Como se observó, en el párrafo anterior, la cantidad de participación ciudadana es más rigurosa en el plebiscito, con relación al referendo. Pero en este punto hay que hacer una obligada aclaración. Para la refrendación de los acuerdos paz no se hizo uso del plebiscito ordinario que establecen las leyes 134 de 1994 y 1757 de 2015, ni tampoco éstas leyes fueron objeto de reforma. Se presentó un proyecto de ley estatutaria que crea un nuevo plebiscito exclusivo para la refrendación de los acuerdos para la terminación del conflicto con las FARC, valga destacar que esta ley superó la revisión constitucional mediante sentencia C-379 de 2016 y fue sancionada por el Presidente de la Republica. Este sui generis mecanismo de participación ciudadana que han denominado "plebiscito para la refrendación del acuerdo final para la terminación del conflicto y la construcción de una paz estable y duradera" tiene unas características o peculiaridades que lo hacen distinto al plebiscito ordinario, que se mantendrá incólume. Esas características especiales son por ejemplo que el "plebiscito por la paz" tiene un umbral de aprobación diferente, esto es, de $13 \%$ del censo electoral a favor del "SI" y que este sea superior al "NO". Inicialmente también se estableció que la participación en estas consultas seria obligatorias y que podían desarrollarse en 3 tres días de un fin de semana, pero estos dos puntos fueron retirados del texto del proyecto de ley.

Así pues, respecto al tópico de la refrendación en el presente proyecto nos acogemos a la tesis planteada por la Corte constitucional en la Sentencia C-379 de 2016, aunque discrepamos de la constitucionalidad que decidió darle este tribunal al porcentaje del umbral de aprobación (13\% para el Sí ), puesto que esta cantidad no representa una verdadera legitimidad popular y comparándolo con el plebiscito de 1957 prácticamente 
se exige la misma cantidad de participación, siendo que para esa fecha Colombia poseía menos de la mitad de población que tiene ahora.

\subsection{EN LO ATINENTE AL MECANISMO DE IMPLEMENTACIÓN}

El gobierno de Colombia terminó un acuerdo definitivo para la terminación del conflicto y la construcción de una paz estable y duradera, con la organización armada de las FARC, respecto a cinco puntos generales: $1^{\circ}$ Desarrollo rural; $2^{\circ}$. Participación en política; 3. Drogas ilícitas y narcotráfico; $4^{\circ}$.Sometimiento a la justicia y Tratamiento a víctimas; 5. Condiciones para el fin del conflicto. Estos acuerdos para ponerlos en marcha, conforme a lo trazado por el ejecutivo, debieron superar dos etapas importantes. Una es la refrendación, de la que ya se trató en el acápite anterior y otra es la implementación. En este segmento haremos alusión a este último mecanismo, que es posterior a la refrendación, que ya fue realizada ganando por escaso margen la opción del "no".

Cuando se menciona el término "implementación" se hace referencia a la manera en que lo acordado pueda plasmarse en normas jurídicas vinculantes que hagan parte del ordenamiento colombiano y por tanto tengan plena eficacia y aplicabilidad. Para la implementación se han propuesto fórmulas como otorgarle facultades extraordinarias al presidente de la república para que expida decretos que surtan tal implementación; o que sea el mismo congreso el que haga lo propio mediante ley, lo que implica el retardo tradicional del trámite legislativo, o que se cree una comisión legislativa especial que mediante un procedimiento expedito establezca las normas que den forma jurídica a los acuerdos, que es lo que se ha dado en llamar "el Fast tract" - que dicho sea de paso lo Corte constitucional mediante sentencia C-332 de 2017, limitó este instrumento expedito-.

Sea preciso aclarar el uso que se da a la palabra "implementación" en la ley 1745 de 2014, en la que se menciona en su artículo $1^{\circ}$ que: "Los referendos constitucionales que sean necesarios para la implementación de un Acuerdo Final para la terminación del conflicto armado estarán sujetos, en especial, a las reglas contempladas en la presente ley". Como puede observarse mediante esta ley se contempla "implementación de un acuerdo", pero no se está haciendo un uso antitécnico de la expresión pues como ya se ha mencionado la palabra adecuada seria refrendación, que es la aprobación popular frente a una determinada decisión. Por otro lado, cuando se expidió esta ley, se llegó a pensar que el mecanismos que se usaría para refrendar los acuerdos sería era el referendo, y por siguiente el congreso se anticipó con esta ley. Agregando que lo más importante que modifica la ley 1745 de 2014 es que estos referendos para refrendación de acuerdos finales de terminación de conflictos, pueden celebrarse incluso en los mismos días de otros actos electorales. Ahora, esta ley quedó como una buena intención pues, no se usó el referendo para el acuerdo con las FARC-EP, pues suponemos que el gobierno por cálculo y estrategia política, redefinió sus planteamientos y pensó que el mecanismo más conveniente era el plebiscito. 
El mecanismo de la implementación se puede dar desde dos enfoques bien diferenciados. Desde un enfoque de producción normativa y desde un enfoque material de ejecución. En cuanto al primer enfoque, se hace referencia, como ya se ha señalado, en convertir los acuerdos en normas jurídicas vinculantes, y de esto podría resultar una modificación de la constitución, la aprobación de nuevas leyes o la expedición de decretos por parte del presidente de la república.

Pero este primer enfoque puede pasar por varias fases, el acto legislativo 01 de 2016 que contempla un procedimiento especial para la implementación de los acuerdos, que como se dijo fue objeto de revisión por parte de la Corte constitucional en sentencia C-332 de 2017, en la que señaló que se violaba la constitución por no permitir procesos deliberatorios que son la esencia de un órgano colegiado como el congreso. Y esto, es justamente lo que pretendió con el acto legislativo 01 de 2016 que dejaba al congreso en la posición de simple notario.

El acto legislativo 01 de 2016 pretendió la instauración temporal de un procedimiento expedito para la implementación de los acuerdos, con el propósito de agilizar y garantizar el trámite legislativo, dando un periodo de seis meses, prorrogable incluso por seis meses adicionales al órgano implementador.

También el presidente de la republica puede expedir decretos para reglamentar las leyes que se promulguen con ocasión de la implementación o expedir decretos especiales respecto de determinados temas en materia de paz, dentro de la órbita de competencia que otorgue el acto legislativo.

Ahora la implementación implica la aprobación de una serie de leyes, que constituirán todo un paquete legislativo relativo a los aspectos contenidos en la los acuerdos. Y una discusión, que se dio fue, ¿qué capacidad tiene los congresistas para introducir modificaciones a los acuerdos, cuando estos pasen por el trámite legislativo especial?, Esta cuestión que se planteó porque quedaba la sensación que los congresistas deben aprobar todo tal cual se encuentra en los acuerdos, y eso fue justamente lo que la Corte constitucional esgrimió para declarar la inconstitucionalidad parcial.

En el acuerdo final para la terminación del conflicto y la construcción de una paz estable y duradera entre Gobierno y FARC-EP de fecha 24 de agosto de 2016, se crea adicionalmente una "Comisión de Implementación, Seguimiento y Verificación del Acuerdo Final de Paz y de Resolución de Diferencias" (CSVR) que tiene entre sus múltiples funciones la de constatar que el contenido de todos los proyectos de decreto, ley o acto legislativo que sean necesarios para implementar el Acuerdo Final, corresponda a lo acordado, antes de que sean expedidos o presentados, según sea el caso, por Presidente de la República.( Acuerdo final, 2016 pg. 175) Como puede observarse, en el acuerdo se prevé la creación de organismo que esté vigilante que 
durante la implementación no se consagren disposiciones que consagren aspectos distintos de los establecidos en los acuerdos.

Una de las primeras normas que fue objeto de implementación es la relativa a la amnistía y los indultos que se deben otorgar a los miembros del grupo de las FARC-EP, la cual por principio de unidad de materia, no debe ir unida en un mismo cuerpo, por ejemplo con normas sobre desarrollo rural, por lo que es necesario el llamado "paquete legislativo". Otras normas con prelación también serán: El Acto legislativo de incorporación de la Jurisdicción Especial para la PAZ, Ley de aprobación del Acuerdo Final, según acuerdo de 11 de mayo de 2016, Acto legislativo y normas de organización sobre la Unidad de Búsqueda de Personas dado por desaparecidas en el contexto y en razón del conflicto armado.

A la implementación quisieron establecerle como base fundamental sobre la cual debió cimentar el resto de normas jurídicas, que a los pactos se les otorgue la categoría de "acuerdo especial". Esto según lo establecido en el Acuerdo de 11 de mayo de 2016 en Gobierno y FARC- EP, según el cual el Acuerdo Final debió ser firmado como Acuerdo Especial del artículo 3 común de las Convenciones de Ginebra y depositado tras su firma ante el Consejo Federal Suizo en Berna. A continuación, por el Presidente de la República se efectuará una declaración unilateral del Estado ante las Naciones Unidas comunicando dicho Acuerdo Final y solicitando la incorporación del mismo a un documento del Consejo de Seguridad de las Naciones Unidas en los términos establecidos en el Acuerdo de fecha 11 de mayo de 2016. Esto debió implicar que los acuerdos hagan parte del Bloque de constitucionalidad, porque se les da tratamiento de un tratado internacional. Aspecto este, que fue muy discutido, desde el punto de vista de sus efectos, por la forma en que debe abordarse estos acuerdos a la hora de surtir el trámite legislativo de la implementación y al final no pudo establecerse como un acuerdo especial, en la forma que inicialmente se había pretendido.

En otro enfoque a que se hará referencia es al material de ejecución, que hace alusión a la parte material de actuación, esto es, todo el conjunto de actividades, acciones, desarrollos operacionales y logísticos que se deben llevar a cabo para poder en práctica el grueso de disposiciones jurídicas que previamente fueron el documento del acuerdo final, además de su evolución y medición con indicadores claros. Para los efectos de este enfoque puede señalarse varios puntos:

Se realizará un Documento CONPES sobre la implementación, pero ya desde el punto de vista de la puesta en práctica de las normas que lo implementa. (Acuerdo Final, 2016 pg. 172), habrán planes cuatrienales en el plan de desarrollo de los nuevos Gobiernos, (los planes de desarrollos deben tener un capitulo que aborde el tema de la implementación de los acuerdos) y un plan plurianual de inversiones que se incorporará al plan de desarrollo nacional actual. 
Por otra parte para hacer seguimiento operativo de los acuerdos, las partes deciden soportarse en la capacidad técnica del Instituto Kroc de Estudios Internacionales de Paz de la Universidad de Notre Dame de los Estados Unidos, el cual Diseñará la metodología para identificar los avances de los acuerdos, Aportará las buenas prácticas y experiencias para un seguimiento efectivo a la implementación de los acuerdos, Construirá con rigor metodológico un modelo de evaluación y seguimiento que permita medir el cumplimiento de los acuerdos, lo suficientemente preciso y que permita en tiempo real, la toma de decisiones y los ajustes, todo ello en el marco de una lógica de mejoramiento continuo de las capacidades de ejecución en la construcción de la paz.

\section{CONCLUSIONES}

De manera conclusiva, nos referiremos a los dos mecanismos objetos de estudio en esta investigación, haciendo las conclusiones en primer lugar respecto a la refrendación. Así pues, sostenemos que pese a que un mecanismo de refrendación para los acuerdos para la terminación del conflicto con las FARC, a luz de la normativa legal y constitucional vigente no es de obligatoria celebración, el gobierno se ha comprometió con su realización. Pero más allá que una deuda de compromiso moral o personal del jefe del ejecutivo, como pareciera ser su empeño. La refrendación debió darse porque esta se traduce en un mandato ciudadano que da total legitimidad democrática a los acuerdos alcanzados, para que sigan el curso posterior de su implementación, además porque es una exigencia del artículo $2^{\circ}$ constitucional, cuando señala que todos tenemos derecho a participar en las decisiones que nos afectan, asimismo, la refrendación permite que diferentes grupos de la población que no pudieron participar en las negociaciones, como cierto sector de víctimas o grupos étnicos se puedan pronunciar desde sus bases más populares.

Lo siguiente que se analizó fue el mecanismo idóneo para su puesta en práctica. Entre la amplia gana que puede ofrecer el ordenamiento jurídico colombiano consideramos que un "plebiscito light" con un umbral más reducido fue conveniente, además de ser practico. El plebiscito actual exige un $50 \%$ del censo electoral vigente, lo cual conociendo nuestra realidad de participación electoral es un exabrupto, teniendo en cuenta que la abstención histórica en Colombia es ligeramente superior a ese $50 \%$. Por tanto una fórmula que establecería una cifra menor es más razonable. Quizá la cifra establecida en el proyecto de ley de un $13 \%$ mínimo por el "SI", siempre que no sea superada por el "NO" fue un tanto baja y se debió ser más proporcionados, en el sentido de tampoco ir a los extremos de que pueda aprobarse una decisión política con una escasa participación, puesto que esto también le resta verdadera legitimidad. Debió establecerse un umbral, tanto para el plebiscito por la paz como para el plebiscito ordinario en una proporción más equilibrada, como la tiene el referendo del $25 \%$ del censo electoral como umbral de participación, puesto que con relación al mecanismo plebiscitario de la ley 134 1994, que nunca se ha ejercitado en vigencia de la constitución de 1991, es impracticable con esas proporciones que tiene hoy, del $50 \%$ del censo electoral. 
A la postre, no aconsejamos el uso del referéndum para este tipo de procesos porque implicaría mayor desgaste para un proceso de paz cualquiera que fuere. Tendría que someterse al trámite de una ley, donde los debates serian interminables para aprobar la formulación de las múltiples preguntas que se exigen, preguntas que quizá gran cantidad de colombianos no logren comprender. Mas el tiempo que implica la revisión de la Corte Constitucional y Con el riesgo latente que se repita la historia del referendo de 2003 en el gobierno de Uribe que solo una pregunta de 18 salió victoriosa y el resto no superó el umbral. Los electores, colombianos de a pie en sus afanes de cada día, prefieren un tarjetón practico para votar y no un cuestionario con tecnicismo de justicia transicional, reforma agraria o mecanismos de reaparición integral víctimas.

En cuanto a la implementación de los "acuerdos final para la terminación del conflicto y la construcción de una paz estable y duradera", puede resumirse que sus bases quedaron sentadas en el acto legislativo No 01 de 2016, que trajo la polémica propuesta de convertir los acuerdos en un acuerdo especial según los artículos 3 común a los convenios de Ginebra, situación ya descartada. Desde el punto de vista del enfoque material de ejecución, también es discutible lo que concierne a la inclusión, hasta cierto punto obligatorio, en el plan de desarrollo nacional, que a la vez implica la armonización de los planes de desarrollo territoriales.

\section{REFERENCIAS BIBLIOGRÁFICAS}

Acto legislativo 01 de 2016, Republica de Colombia.

Acuerdo final (2016). Para la terminación del conflicto y la onstrucción de una paz estable y duradera. 24 agosto de 2016.

Altman, David (2010). Plebiscitos, referendos e iniciativas populares en América Latina: ¿mecanismos de control político o políticamente controlados?. Revista Perfiles latino americanos. vol.18 no.35 México ene./jun. 2010.

Ámbito jurídico (2016). ¿Es necesaria la refrendación popular de los acuerdos de paz? foro del Ciclo de conversatorios nacionales y regionales sobre el proceso de paz en Colombia. Universidad Sergio Arboleda.

Bejarano, Ramiro (2016). Plebiscito Vs constituyente. ¿Cuál es mejor para refrendar?. Periódico el Heraldo. 2016 --02 - 15.

Misión de observación electoral MOE (2013). Rutas jurídicas: Refrendación ciudadana de acuerdos de paz. Oficina jurídica Moe, Bogota. 
Revista Semana (2015). Refrendación del proceso de paz: ¿sí o no?. 20 Enero 2015. Versión electrónica.

Ley 134 de 1994. Congreso de la Republica de Colombia. Por la cual se dictan normas sobre mecanismos de participación ciudadana.

Ley 1757 de 2015. Congreso de la Republica de Colombia. Por la cual se dictan disposiciones en materia de promoción y protección del derecho a la participación democrática.

Hernández Becerra, Augusto (2003). Convocatorias al pueblo en Colombia. Revista Credencial Historia. Edición 159 (Bogotá - Colombia). Marzo de 2003.

León, Juana (2015). El rompecocos de la refrendación. Revista electrónica la Silla Vacía. Enero 2015.

Sentencia C-141 de 2010. Corte constitucional Colombiana. M.P. Humberto Antonio Sierra Porto

Sentencia C - 1121 de 2004. Corte constitucional Colombiana. M.P. Clara Inés Vargas Hernández

Sentencia C - 379 de 2016. Corte constitucional Colombiana. M.P. Luis E. Vargas

Uprimy, Rodrigo (2013). La refrendación democrática de la paz. UN periódico. Universidad Nacional de Colombia. Bogotá, mayo 2013

Zovatto, Daniel (2010). Las instituciones de la democracia directa a nivel nacional en América Latina. Balance comparado: 1978-2010. Revista de sociología, № 24 (2010) pp. 87-124 